\title{
Inferior Alveolar Nerve Radiofrequency Ablation For Refractory Trigeminal Neuralgia: A Case Report
}

\author{
Neal A. Shah, MDㄹ, Chandni B. Patel, BS ${ }^{2}$, Ankur A. Patel, DO ${ }^{3}$, and Devang Padalia, MD
}

Trigeminal neuralgia, also known as tic douloureux, is a disorder characterized by pain in the distribution of the trigeminal nerve. Chronic pain secondary to this condition can have a significant negative impact on a patient's quality of life. We present an educational case of refractory trigeminal neuralgia responsive to a novel painalleviating procedure. An 80-year-old man with recurrent trigeminal neuralgia presented with episodic pain refractory to multimodal pharmacologic treatment, as well as interventional pain procedures. Radiofrequency ablation (RFA) to the mandibular and maxillary branches of the trigeminal nerve was attempted, but deemed unsuccessful. In an attempt to relieve the patient's pain in the mandibular region, an inferior alveolar nerve block with radiofrequency ablation was performed. The patient reported a significant longterm reduction of his pain and improved ability to perform activities of daily living.

Key words: Trigeminal neuralgia, tic douloureux, radiofrequency ablation, trigeminal nerve, inferior alveolar nerve
Trigeminal neuralgia (TN) is a disorder typically characterized as episodic, unilateral pain in the distribution of the trigeminal nerve, most commonly the maxillary (V2) or mandibular branches (V3). Although descriptions of trigeminal neuralgia have been around for centuries, the first documented case was not until the late $1700 \mathrm{~s}$ (1). The prevalence is estimated to be 1 to 2 per 100,000, with women affected more than men at a 3:2 ratio. Research has shown that $\mathrm{TN}$ is commonly associated with underlying arterial hypertension and multiple sclerosis. The most common etiology of TN is vascular compression of the trigeminal nerve root, which results in focal demyelination and aberrant neural discharge $(2,3)$. Symptoms are often debilitating and affect patients' activities of daily living; hence prompt recognition is necessary, as is initiation of

From : ${ }^{1} \mathrm{H}$. Lee Moffitt Cancer Center and Research Institute, Tampa, FL; ${ }^{2}$ Edward Via Virginia College of Osteopathic Medicine, Blacksburg, VA; ${ }^{3}$ NewYork-Presbyterian Hospital/Columbia \& Cornell, New York, NY

Author for correspondence: Devang Padalia, MD

Address: H Lee Moffitt Cancer Center and Research Institute,12902 USF

Magnolia Drive Tampa, FL 33612

E-mail: Devang.Padalia@moffitt.org appropriate pharmacologic and surgical interventions to manage this debilitating and painful condition.

\section{CASE PRESENTATION}

An 80-year-old man with no significant past medical history presented to the interventional pain clinic with a chief complaint of recurrent episodes of right-sided facial pain for 8 years. Episodes of pain were described as sharp, localized right-sided facial pain that originated under the tongue and radiated outwards towards the base of the jaw, medial border of the ear, and zygomatic process. The patient reported that the pain was triggered by eating, chewing, shaving, and speaking. These episodes occurred up to 20 times per day causing significant impairment to his daily activities and quality of life. The patient denied any recent craniofacial trauma, recent dental procedures including dental extractions, and no history of herpes zoster infection. He denied any dizziness, focal weakness, headaches, vision changes, or ataxia. Based on the clinical history and high suspicion of $\mathrm{TN}$, magnetic resonance imaging (MRI) of the brain was completed, which ruled out any neurovascular compression or space-occupying lesion or mass. A 
trial of oral pharmacologic agents including carbamazepine, gabapentin, and verapamil was initiated. This regimen was initially effective, but the patient became refractory to this treatment and continued to have intermittent episodes of pain. Furthermore, the patient had disequilibrium and cognitive side effects from these pharmacologic agents. The patient was not interested in trying other pharmacologic or invasive surgical therapies, but agreed to a diagnostic trigeminal nerve block of the right V2 and V3 nerve branches. This block provided the patient greater than $80 \%$ pain relief and he was therefore deemed an appropriate candidate for radiofrequency ablation (RFA) of these nerves. The RFA procedure was performed by skilled interventionalists; sensory testing prior to ablation indicated concordant pain within the distribution of the trigeminal nerve, emphasizing correct placement of the RFA electrode. However, the patient's symptoms did not resolve. A repeat RFA procedure with sensory testing was performed, but the patient reported having continued pain in the distribution of the lower jaw and mandible. In addition, the patient developed right-sided tongue numbness and intermittent slurring of his speech. Since the patient had minimal relief from the usual pharmacological and interventional therapies, a novel right inferior alveolar nerve block with RFA was proposed.

The inferior alveolar nerve block was conducted by initially identifying the coronoid notch, located at the region of greatest concavity on the anterior border of the ramus, and the deepest part of the pterygomandibular raphe. The needle insertion site was identified 3 quarters the distance from the coronoid notch to the deepest part of the pterygomandibular raphe (Fig. 1) and approximately 6 to $10 \mathrm{~mm}$ above the occlusal plane of the lower teeth. A 25-gauge, 1.5 -inch needle was slowly advanced transorally until reaching the mandible, after which the needle was slightly withdrawn. After the needle location was confirmed with fluoroscopy (Fig. 2) and there was negative aspiration of blood, $5 \mathrm{cc}$ of $0.25 \%$ bupivacaine was injected in a fanned-out pattern. The diagnostic block was deemed successful with complete pain relief for over 8 hours; therefore, the patient was brought back for RFA of the inferior alveolar nerve. Using the same technique, a $100-\mathrm{mm}$ RFA needle with a 5-mm active tip was advanced towards the nerve. Sensory stimulation of the nerve was concordant to the patient's area of pain at $0.5 \mathrm{~V}$, 1 millisecond pulse duration, and a stimulation rate of $50 \mathrm{~Hz}$. Motor stimulation at $1.7 \mathrm{~V}$, a 1 -millisecond pulse duration, and a $2-\mathrm{Hz}$ stimulation rate produced movement of the mylohyoid and the anterior belly of the digastric muscle. After testing, RFA of the nerve was conducted for 90 seconds at 80 degrees Celsius. The patient had no postprocedure complications and did not report any muscular weakness. At the oneday, one-month, and 6-month follow-ups, the patient reported greater than $70 \%$ relief of his pain and was very satisfied with the results.

\section{DISCUSSION}

The trigeminal nerve is the major sensory nerve for the face and the major motor nerve for the muscles of mastication. The nerve emerges off the brainstem at the level of the midpons and continues through the prepontine cistern and Meckel's cave to the trigeminal ganglion (4). From the trigeminal ganglion, the nerve splits into 3 major divisions: ophthalmic (V1), maxillary (V2), and mandibular (V3). This patient had pain primarily along a branch of the mandibular nerve called the inferior alveolar nerve. This nerve branches off the mandibular nerve at the level of the mandibular ramus and continues inferiorly into the mandibular foramen before it gives rise to the mylohyoid nerve, a motor branch innervating the mylohyoid muscle and anterior belly of the digastric nerve. The inferior alveolar nerve also gives rise to 2 sensory branches, the mental and incisive nerves. The mental nerve provides sensation to the anterior chin, lower lip, and buccal gingiva of the anterior mandibular teeth. The incisive nerve provides sensation to the first premolar, canine, and incisor teeth and their buccal mucosa.

TN is a chronic pain condition that is described as unilateral, intermittent, sharp, electric-like pain in the distribution of one or more branches of the trigeminal nerve, with the maxillary branch being the most commonly affected. Episodes are typically triggered by nonpainful stimuli including light touch, brushing, chewing, talking, and smiling. The diagnosis of primary TN is a clinical diagnosis with paroxysms of pain in the distribution of the trigeminal nerve with an unknown cause. When there is a clinical suspicion of $\mathrm{TN}$, it is imperative to search for potential causes. Secondary causes of TN are more common in those 


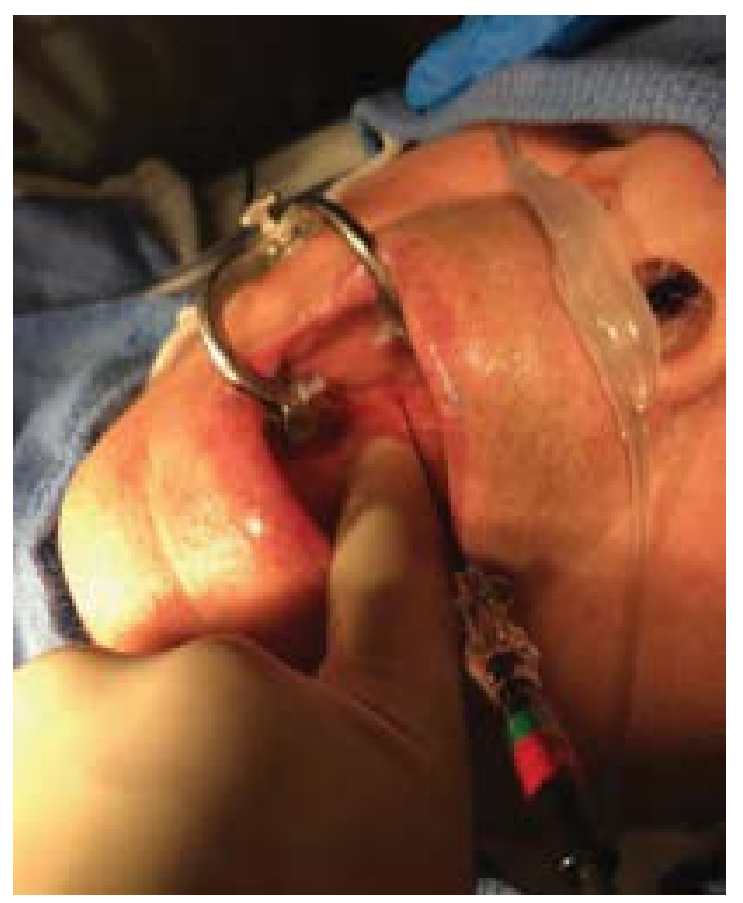

Fig. 1. Needle insertion site at the height of the coronoid notch into the deepest part of the pterygomandibular raphe.

who have bilateral symptoms, age less than 40 years old, a history of multiple sclerosis, and/or hypoesthesia or hyperalgesia in the affected trigeminal region. Common secondary causes of TN include multiple sclerosis plaques, intracranial masses, acute herpes zoster, postherpetic neuralgia, and trauma (2). Even though these clinical features can help clinicians suspect secondary causes of TN, the absence of these features does not definitively rule out the possibility of secondary causes. Neuroimaging, in particular an MRI of the brain with and without contrast medium, is recommended to rule out any secondary causes; however, surgery is often required to definitively rule out vascular compression that may not be observed on neuroimaging. Additionally, the International Headache Society has developed the third edition of The International Classification of Headache Disorders (ICHD-3) (5), which outlines the diagnostic criteria for TN; these include:

1. Recurrent episodes of unilateral facial pain in the distribution of one or more divisions of the trigeminal nerve;

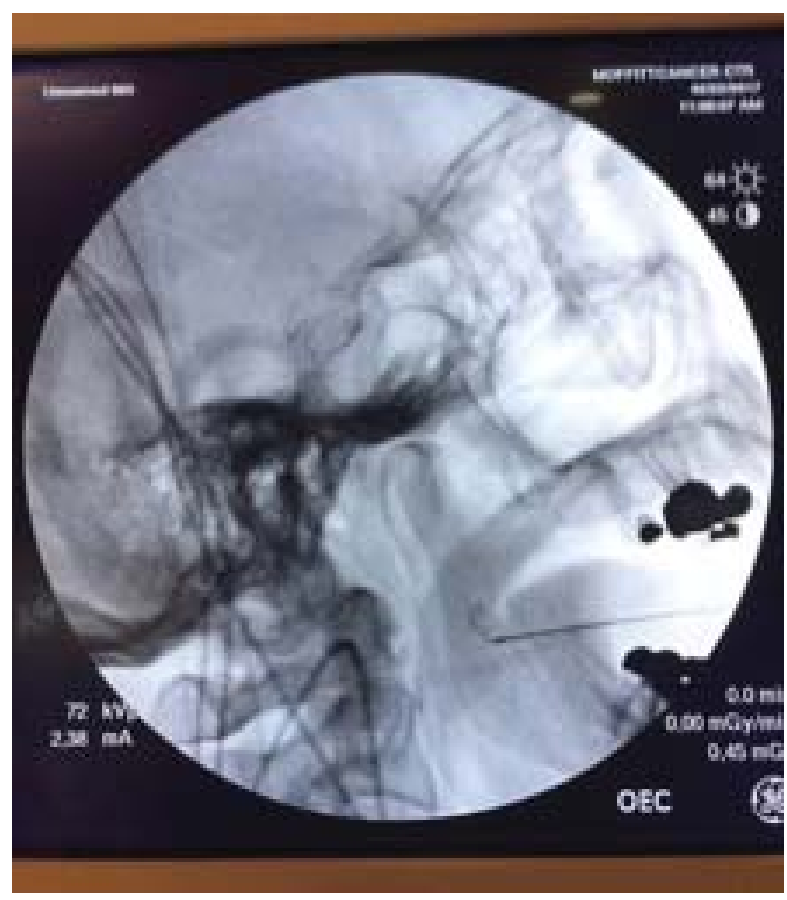

Fig. 2. Confirmed needle placement with fluoroscopy.

2. Pain that is characterized as brief (lasting a second to 2 minutes), severe intensity, and shocklike, sharp quality pain;

3. Triggered by a noxious stimulus;

4. Pain is not characterized any better by other ICHD-3 diagnostic criteria.

For our patient, neuroimaging for any space-occupying masses, lesions, or vascular compression of the trigeminal nerve and clinical presentation fulfilled the ICHD-3 diagnostic criteria.

Although there are many different causes of TN, $90 \%$ of cases are caused by compression of the trigeminal nerve root by an artery or vein, most commonly the superior cerebellar artery (6). Other causes of trigeminal nerve root compression include acoustic neuroma, meningioma, aneurysm, or arteriovenous malformation (7). The compression leads to an axonal injury resulting in demyelination leading to the symptoms of trigeminal neuralgia (2). TN is primarily 
a clinical diagnosis in which neuroimaging can help differentiate between primary and secondary causes, but is not required for diagnosis. The treatment of $\mathrm{TN}$ is often a challenge; however, a multidisciplinary approach is recommended to provide better care for this population. Conservative management with pharmacologic agents is the first line of treatment. The most effective and first-line pharmacological treatment for this condition is carbamazepine, an anticonvulsant agent. Although the mechanism of analgesia is unconfirmed, it is thought that the blockage of voltage-gated sodium channels stabilize the neuronal impulses associated with TN, providing symptomatic relief. Studies have shown carbamazepine having approximately $80 \%$ initial efficacy in resolving symptoms; however, the side effect profile is often the limiting factor with this medication (8). Other pharmacologic treatments include oxcarbazepine, baclofen, lamotrigine, and pimozide; these are often recommended for those who are unable to tolerate the side effects associated with carbamazepine.

For individuals who fail multiple pharmacologic therapies, as in our case, there are a wide range of interventional therapies available with varying levels of effectiveness that can help treat TN. Microvascular decompression (MVD) surgery is the most effective surgical treatment in providing long-term pain relief in $90 \%$ of patients with TN (8). MVD involves a craniotomy to remove or relocate the superior cerebellar artery that is placing pressure on the root of the trigeminal nerve. This is done by placing a sponge or pad between the trigeminal nerve and the artery to remove the pressure and pulsatile effects of the artery (10). However, there are significant risks for aseptic meningitis, occurring in up to $11 \%$ of patients, and chronic hearing loss, occurring in up to $10 \%$ of patients (11). Even though MVD is an effective treatment option for TN, it is typically recommended for patients younger than 70 years old due to the complications that can arise with the use of general anesthesia (12). For those individuals who are above the age of 70 and/or who have multiple morbid conditions, RFA is the recommended treatment option if adequate relief is obtained from a trigeminal ganglion block. RFA uses high frequency radio waves $(500 \mathrm{kHz})$ to ablate the nervous tissue in the treatment of chronic pain. A study examining the outcomes of using RFA in 1,600 patients diagnosed with TN over 25 years reported $97.6 \%$ of patients having acute pain relief, with $7.7 \%$ of patients having pain recurrence within 6 months (13). At the 5-year follow-up, about $57.7 \%$ of the patients who underwent only a single procedure had complete pain relief and $92 \%$ of patients with either a single procedure or multiple procedures continued to have pain relief (14). The initial pain relief post RFA is comparatively similar to that obtained with MVD. However, with RFA there is a $15 \%$ to $20 \%$ rate of pain reoccurrence in 12 months, which is the lowest recurrence rate among all the percutaneous techniques (15). Although the long-term efficiency of RFA is lower compared to MVD, RFA can be considered the treatment of choice when pharmacologic agents fail, due to the associated lower mobility and mortality rates (16).

Another treatment modality includes percutaneous glycerol injection in which glycerol, a mild neurolytic, is injected into Meckle's cave where the trigeminal nerve ganglion lies. In a long-term follow-up study, $97.1 \%$ of the patients reported immediate success post injection and $81.18 \%$ had long-term success (17). Gamma knife surgery (GKS) is the least invasive non-pharmacologic treatment modality, which uses radiation to target the root of the trigeminal nerve. This slows the formation of a lesion on the nerve and prevents transmission of pain signals to the brain (18). Outcomes and the complication rate with GKS are similar to other ablative therapies, making GKS an attractive modality; however, the delayed onset of pain relief is a significant drawback.

For patients who fail to respond to the initial RFA, a repeat procedure is a recommended first option since RFA is a low-risk, minimally invasive procedure with a high rate of efficacy. If pain persists following repeat RFA, it is imperative for practitioners to reassess the radiation pattern and physical examination findings and consider targeting more specific nerve branches, like in this case the inferior alveolar nerve. Additionally, since other techniques including glycerol ablation, balloon compression, or gamma knife surgery have similar efficacy compared to RFA, these all serve as viable options when treating patients who have failed RFA. If the patient continues to not respond to the various procedures previously mentioned, an invasive MVD should be considered. Even though the MVD provides the lowest rate of pain recurrence 
compared to other treatment modalities, the risks associated with an invasive surgery need to be weighed against the supposed benefits.

Recent case reports have shown success with the use of peripheral nerve stimulation as a treatment of refractory TN (18). Although current studies show promising outcomes, there have not been long-term studies evaluating the use of nerve stimulation for TN. Further research regarding this modality would help clinicians incorporate it in the treatment algorithm for $\mathrm{TN}$ and other chronic pain syndromes.

\section{CONCLUSION}

Trigeminal neuralgia is a debilitating condition that affects the quality of life of many patients. In many patients, pharmacologic and/or interventional treatments can provide great success. However, as outlined in our case, there is a subset of patients who suffer from refractory TN, which can be difficult to manage. To our knowledge, we present the first case report describing RFA of the inferior alveolar nerve for treatment of refractory trigeminal neuralgia. Targeting the inferior alveolar nerve can be a viable option in patients who suffer from refractory trigeminal neuralgia and have had minimal benefits from pharmacological treatments.

Physicians should consider this novel procedure for treatment of refractory cases of trigeminal neuralgia prior to more invasive surgical procedures, which carry an increased chance for severe complications.

Early and effective intervention for patients suffering from this condition can significantly improve their ability to function normally and improve their quality of life. 


\section{REFERENCES}

1. History of trigeminal neuralgia. UT Health San Antonio Web site. www.uthscsa.edu/patient-care/physicians/services/trigeminal-neuralgia/history-trigeminal-neuralgia. Website update 10/31/2017. Date Accessed 11/11/2017.

2. Love S, Hilton DA, Coakham HB. Central demyelination of the Vth nerve root in trigeminal neuralgia associated with vascular compression. Brain Pathol 1998; 8:1-11.

3. Emril DR, Ho KY. Treatment of trigeminal neuralgia: Role of radiofrequency ablation. J Pain Res 2010; 3:249-254.

4. Hacking C, Gaillard F. Trigeminal nerve. Radiopaedia Web site. https://radiopaedia.org/articles/trigeminal-nerve. Date Accessed $11 / 11 / 2017$.

5. Headache Classification Committee of the International Headache Society (IHS). The International Classification of Headache Disorders, 3rd edition (beta version). Cephalalgia 2013; 33:629-808. doi:10.1177/0333102413485658.

6. Hamlyn PJ. Neurovascular relationships in the posterior cranial fossa, with special reference to trigeminal neuralgia. Clin Anat 1997; 10:380-388.

7. Matthies C, Samii M. Management of 1000 vestibular schwannomas (acoustic neuromas): Clinical presentation. Neurosurgery 1997; 40:1-9.

8. Obermann M. Treatment options in trigeminal neuralgia. Ther Adv Neurol Disord 2010; 3:107-115.

9. Bohluli B, Motamedi MH, Heydari M, Heydari M. Drug-refractory trigeminal neuralgia: Treatment via botulinum toxin type $A$. In: Motamedi MH. A Textbook of Advanced Oral and Maxillofacial Surgery Volume 3. London, United Kingdom: IntechOpen; 2016. URL: www.intechopen.com/books/a-textbook-of-advancedoral-and-maxillofacial-surgery-volume-3/drug-refractory-trigeminal-neuralgia-treatment-via-botulinum-toxin-type-a. Date Published 08/31/2016. Date Accessed 11/11/2017.

10. Tew J, McMahon N. Microvascular decompression (MVD). May- field Brain \& Spine Web site. www.mayfieldclinic.com/PE-MVD. htm. Website updated 07/2018. Date Accessed 01/06/2018.

11. Gronseth G, Cruccu G, Alksne J, Argoff C, Brainin M, Burchiel K, Nurmikko T, Zakrzewska JM. Practice parameter: The diagnostic evaluation and treatment of trigeminal neuralgia: Report of the Quality Standards Subcommittee of the American Academy of Neurology and the European Federation of Neurological Societies. Neurology 2008; 71:1183-1190.

12. Eugene, AR. Trigeminal neuralgia and radiofrequency lesioning. Brain (Bacau) 2015; 6:91-96.

13. Kanpolat $Y$, Savas A, Bekar A, Berk C. Percutaneous controlled radiofrequency trigeminal rhizotomy for the treatment of idiopathic trigeminal neuralgia: 25-year experience with 1,600 patients. Neurosurgery 2001; 48:524-532.

14. Trigeminal Neuralgia Treatments. Johns Hopkins Medicine Web site. www.hopkinsmedicine.org/neurology_neurosurgery/centers clinics/trigeminal neuralgia/trigeminal neuralgia treatments.html. Date Accessed 03/16/2018.

15. Gamma Knife Radiosurgery, Radiation, and Side Effects. University of Pittsburgh Schools of the Health Sciences Web site. www.upmc.com/services/neurosurgery/brain/treatments/gamma-knife-radiosurgery/pages/default.aspx. Date Accessed 11/11/2017.

16. Headache Classification Committee of the International Headache Society (IHS). The International Classification of Headache Disorders, 3rd edition. Cephalalgia 2018; 38:1-211.

17. Chen L, Xu M, Zou Y. Treatment of trigeminal neuralgia with percutaneous glycerol injection into Meckel's cavity: Experience in 4012 patients. Cell Biochem Biophys 2010; 58:85-89.

18. Shaparin N, Gritsenko N, Garcia-Roves DF, Shah U, Schultz T, DeLeon-Casasola O. Peripheral neuromodulation for the treatment of refractory trigeminal neuralgia. Pain Res Manag 2015; 20:63-66. 